\title{
DYNAMIC QOS PARAMETER ADAPTATION IN ROUTERS USing A MUlTilayer NeURAl NeTWORK
}

\author{
Irina Topalova \& Plamena Koleva
}
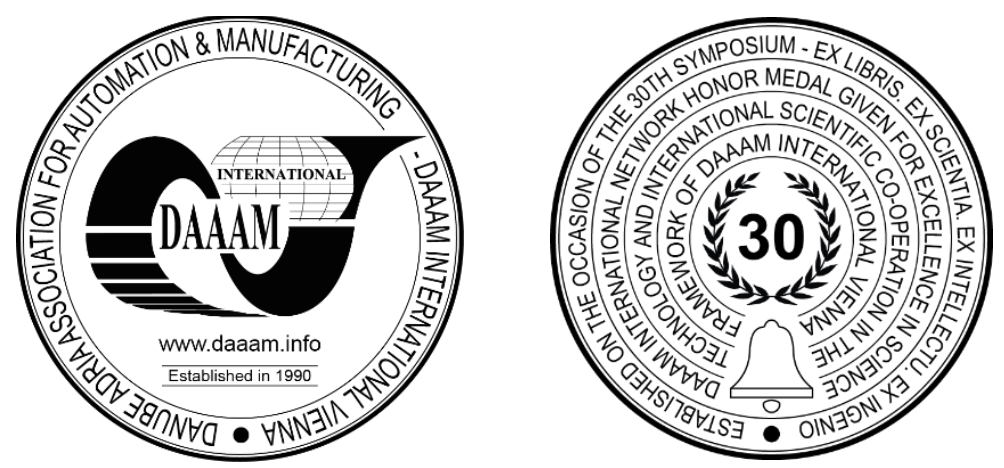

This Publication has to be referred as: Topalova, I[rina] \& Koleva, P[lamena] (2019). Dynamic QoS Parameter Adaptation in Routers Using a Multilayer Neural Network, Proceedings of the 30th DAAAM International Symposium, pp.0207-0214, B. Katalinic (Ed.), Published by DAAAM International, ISBN 978-3-902734-22-8, ISSN 1726-9679, Vienna, Austria

DOI: $10.2507 / 30$ th.daaam.proceedings.027

\begin{abstract}
Practical prevention of network congestion is quality of service (QoS). Connection-oriented protocols, such as a TCP protocol, generally look for packet errors, losses, or delays to adjust the transmission speed. Currently, congestion control and avoidance algorithms are based on the idea that packet loss is a suitable indicator of network congestion. The data is transmitted using the Best Effort service model. When a network uses this model, it provides data without guaranteeing reliability and delay. However, these algorithms and the best effort service model are not suitable for applications that respond to delays or packet loss. WRED is implemented on the core routers of a network. The edge routers assign packets with IP priorities when packets enter the network. A neural network is trained to automatically adapt new end users to the quality of service policy, already set by other end-users and accepted by the intermediate routers. The obtained results show that the automated adaptation of the Quality of Service parameters to the already set ones, is possible for the intermediate routers, and the positive cons equences of applying such a method are discussed.
\end{abstract}

Keywords: traffic congestion; Quality of Service; early detection; queue management; neural network.

\section{Introduction}

An exchange of data occurs through constant transmission of packets between routers, between a router and a computer, or between different smart devices. Therefore, there is the case that data packets may be lost. The problems can be for various reasons. From the point of view of the sender and receiver, these problems can be a delay, an error or the delay of the end-to-end transmission. One of the most important points is the correct configuration of the core router in the network. In this research a method is proposed for dynamically QoS parameter adaptation in routers using a multilayer neural network. The communication between the routers, the computer and the neural network is realized by an application of an API, using the programming language Python. The reason for creating this work is the possible overload control in a network with more than three routers, if data packets are omitted and the information does not reach the end devices. The proposed paper is organized as follows. Section 2. describes related to the research works. Section 3. describes the proposed method and the neural network topology. Section 4. gives the experimental results. The conclusion closes the article. 


\section{Related Works}

The authors Samira Chabaa, Abdelouhab Zeroual and Jilali Antari describe in their work the development of an artificial neural network (ANN) model based on the multi-layer perceptron (MLP) for analyzing internet traffic data over IP networks. They used an artifitial neural network to measure a time series data for network response evaluation [1]. This work helped for decisioning the training algorithm for the neural network. The used training method is backpropagation.

The authors A.Macura, E. Missioni and B. Marcovic are implementing a specific algorithm for synchronization based on a selection of relevant QoS parameters, in order to enable realization of the desired multimedia applications. The requested QoS presents one of the important elements of the design and implementation of multimedia networks. The authors analyse the parameters of importance for multimedia communications, but they limit/focus themselves only on the specifics of multimedia communications [2]. In other article is described the exploration the interest of computational intelligence tools in the management of heterogeneous communication networks, in order to predict network congestion and failures in the network that may lead to degradation of the quality of offered services [3]. There are used two different applications based on neural and neuro-fuzzy systems for Quality of Service (QoS) management in next generation networks. Referring mainly to the article "Control of Traffic Congestion with Weighted Random Early Detection and Neural Network Implementation", the proposed method there is extended in this work to use an applicational programming interface, which corresponds with the neural network and the interface of the routers. The results from their study show that the automated adaptation of the Quality of Service parameters, is possible for the intermediate routers and have positive consequences for applying them in real systems [4].

\section{WRED Functionality}

Currently, Transmission Control Protocol (TCP) congestion control and avoidance algorithms are based on the idea that packet loss is a suitable indicator of network congestion. The data is transmitted using the Best Effort service model [5]. When a network uses this model, it provides data without guaranteeing reliability and delay. However, these algorithms and the best effort service model are not suitable for applications that respond to delays or packet loss (e.g., interactive data traffic including telnet, web browsing and transmission of audio and video data). Weighted Random Early Detection (WRED) using Explicit Congestion Notification (ECN) solves this problem [6]. WRED allows early detection of congestion and offers the ability to handle multiple traffic classes. WRED can selectively discard lower priority traffic as the router experiences congestion and provides different capabilities for many classes of service. It also protects against global synchronization. The global synchronization occurs as waves of the dam, followed by periods when the link is underutilized. For these reasons, WRED is useful for all output interfaces or routers where congestion is expected. WRED is implemented on the core routers of a network. The edge routers assign packets with IP priorities when packets enter the network. In WRED, core routers then use these priorities to determine how many types of traffic should be handled. WRED offers separate thresholds and weights for different IP priorities so that the network can provide different quality of service for different types of traffic. During traffic congestion, standard traffic may fall more frequently than premium traffic [7].

When a packet arrives, the following events occur:

- The average queue size is calculated.

- If the average is below the minimum threshold of the queue, the incoming packet is queued.

- If the average is between the minimum queue threshold for this traffic type and the maximum threshold for the interface, the packet is either discarded or queued depending on the packet drop probability for that traffic type.

- If the average queue size exceeds the maximum threshold, the packet is discarded.

The weighted random early detection (WRED) algorithm is implemented on the central router. In this way, it can decide which packets from the queue should be dropped and which should not [8]. Routers RR1 and RR2 contain only the classification of the data packets. The central router is bound by a network bridge with the real computer on which the program for the simulation is running.

New router has also implemented the WRED algorithm but with different queuing threshold size values. The goal is that it is taught from the neural network. An end-to-end QoS list is created with different types of traffic classes that use the Cisco IOS Differentiated Services (DiffServ) features.

\section{Mark Probability Denominator (MPD)}

It has become clear that WRED as an algorithm avoids data congestion. For this process, the algorithm also needs other metrics, e.g. the probability of the failed packages. WRED ensures the probability of each class specified how many packets should fail. The principle is the following: The higher the priority of a data class, the smaller the likelihood that a packet will fail. The random - detect command can be used to define the priority value, the minimum and maximum threshold for a data transfer quantity, and the Mark Probability Denominator. These values determine the behavior of this algorithm in each queue [9]. 
MPD (Mark Probability Denominator) can be defined as follows: The probability that a packet should be dropped is decided by the mark probability denominator. It is the fraction of packets dropped when the average queue size reaches the maximum threshold of the queue. Figure 1 describes exactly the process as it is.

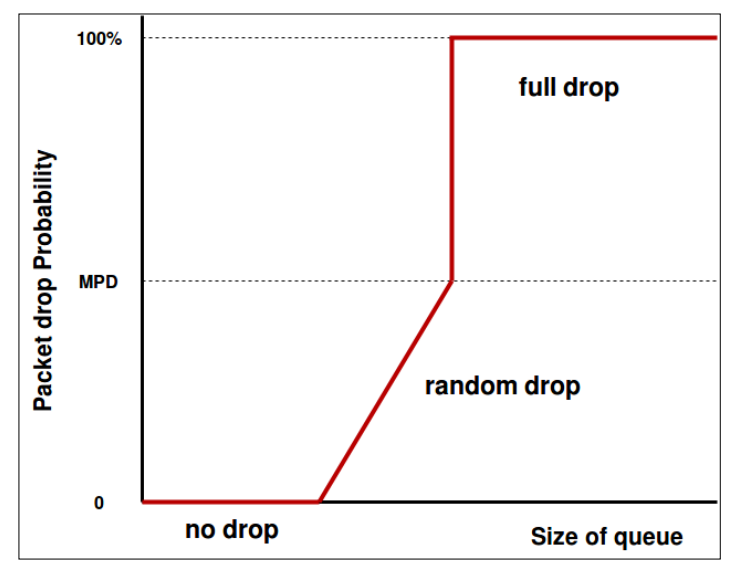

Fig. 1. Process for avoiding data congestion in WRED

The following process is used:

- The average queue is calculated.

- If the average queue threshold is less than the minimum, the incoming packet is queuing (no drop).

- If the average queue threshold is between the minimum and maximum thresholds for this interface, the packet will fail in relation to the packet drop probability (random drop).

- If the average queue threshold is greater than the maximum threshold, the packet will fail (full drop).

\section{Proposed Method for QoS parameter adjustment}

In this study, we apply the WRED method for QoS in a network having end routers, a central/backbone router and an ad-hoc "New router". The first task is to force the new added router to comply with the QoS requirements, which were pre-set in the central router. For this we propose a Neural Network, intended to work in the central router, aiming to adjust the parameters of the "New" to the existing ones. The second task is to propose a method for appropriately determining the average queue and the min-threshold in the central router, considering the current average queue of the added "New router".

\subsection{Topology}

The proposed method describes a topology of Cisco routers from "Series 2900". As shown in Figure 2 there are four routers: RR1 (Remote Router 1), Central Router, RR2 (Remote Router 2) and New Router (a new router). There are two computers in addition, namely PC1 and PC2. On both computers is installed the operating system Linux with the distribution Tiny Core OS. They serve to analyze the data volume. The focus is on the network protocols HTTP and SMTP. The reason for this choice is the larger data flow and the subsequent good analysis of the data volume. An MLP (multilayer linear perceptron) neural network is responsible for adapting the QoS policy. The central router is connected to the three routers through serial ports. It is in one network with the router RR1 through Serial Interface 1/0. A network bridge connects two separate computer networks. This bridge enables communication between the two different networks and offers the possibility to work as a single network. They still extend local area networks and allow coverage of larger physical areas that the LAN (Local Area Network) can otherwise reach. For this work, the configuration of the network bridge is used by the operating system Linux.

An application programming interface (API) is responsible for extracting the necessary part of data from the router and passing it to the neural network of the QoS parameters. This program is located on the computer which is connected directly to the central router. After training the data in the neural network, the result is edited and returned to the central router via SSH connection. The processing of the data is done by a program, written on Python.

The proposed method uses a software for simulating the whole process. The software GNS3 emulates, configures, tests and troubleshoots virtual and real networks. With GNS3 can be ran a small topology that only consists of a few devices on the computer. Bridges lack the intelligence of network routers: Bridges do not understand the concept of remote networks and cannot dynamically redirect messages to other sites, but only support an external interface. 
For this work, the configuration of the network bridge was used by the operating system Linux. In order to work properly this networking, three smaller programs have been created. First the connection with the central router must be established. The following command was executed in the terminal:

\section{brctl addif virbr0 enp0s 25}

With brctl, the Ethernet bridge configuration in the Linux kernel is set up, maintained and checked. With the command brctl addif <name of the network bridge> <name of the interface>, the interface enp0s25 becomes a port of the bridge virbr0. This means that all data frames received on enp0s 25 are processed as if they arrive in the network bridge virbr0.

After creating the connection with the virtual network bridge, the routing must be configured and all networks, used in the GNS3 program, must be written on the real computer. Every computer, connected to a network, needs some sort of routing instructions for network TCP / IP packets as they leave the local host. By default, every real machine that has a network connection has a default gateway. But the real computer does not know about the virtual networks. Therefore these networks have to be described explicitly. The following commands were executed in the terminal:

ip r a 192.168.124.0/24 via 192.168.122.3

ip r a 192.168.125.0/24 via 192.168.122.3

ip $r$ a 192.168.126.0/24 via 192.168.122.3

ip $\operatorname{r}$ a 192.168.127.0/24 via 192.168.122.3

The ip command can be used to view or edit the routing, devices, policy routing, and tunnel. The parameters $r$ and $a$ mean that you want to add the following network to the routing table. The parameter $r$ stands for Routing and the parameter $a$ stands for Add. The final parameter via 192.168.122.3 describes from which IP address the data packets should be transferred. The executed commands add in the routing table of the real computer the networks: 192.168.124.0/24, 192.168.125.0/24, 192.168.126.0/24, 192.168.127.0/24.

But that is not enough for this work. On the computers PC1 and PC2 the monitoring program "iperf3" is needed to perform the correct test for data transfer. For this purpose, a small program is created to configure the firewall of the real computer:

iptables -I FORWARD - i virbrO -j ACCEPT

iptables -I FORWARD -j ACCEPT

iptables -t nat -I POSTROUTING! -d 192.168.120.0/20 -j MASQUERADE

The program iptables is a management program for IPv4 packet filtering and NAT. It sets up, manages and verifies the IP packet filter rules tables in the Linux kernel. A firewall rule specifies criteria for a package and a destination. If the packet does not match, the next rule in the chain is checked. If not, the next rule is given by the value of the target. This can be the name of a custom string or one of the special values ACCEPT, DROP, QUEUE or RETURN. ACCEPT means to let the packet through. DROP means dropping the package to the ground. QUEUE means to forward the package to the user space.

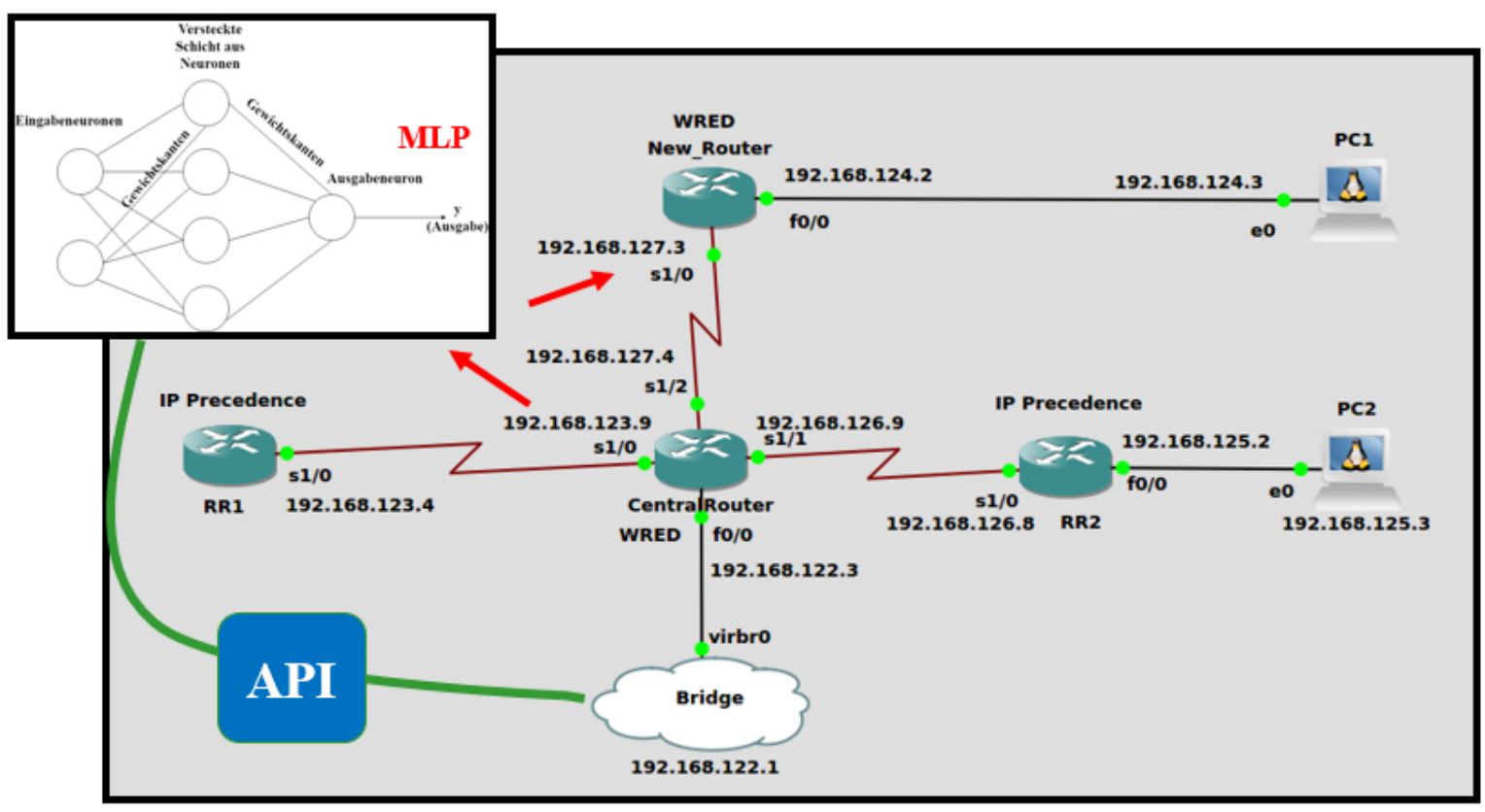

Fig.2. Topology of the proposed system 
The goal of this configuration is to give the ability the two machines from the GNS3 software to communicate with the real Internet and between them. This alleviates that the virtual machines transmit data packets outside the program to the real world.

\subsection{Neural Network strategy}

It was mentioned, that the router "New Router" (see Fig.1) should be taught, how many packets it should discard. Therefore, an API (Application Programmable Interface) communicates with a neural network of type MLP (multi-layer perceptron) on a real computer. This neural network takes the data from the queues with the data packets and trains their values. The result is the value of the Mark Denominator (MD). The goal for the new router is to inherit the value of the neural network trained Mark Denominator (MD) when it is bound to that topology.

In the record, the input data, $\mathbf{x}$, is a $3 \times 3$ matrix. The output data, $\mathbf{y}$, is a $3 \times 1$ matrix. Each element in the matrix $\mathbf{x}$ must be multiplied by a corresponding weight. Then it is added together with the result of each neuron in the hidden layer. Random values of weights are used in the applied neural network. First, the result of the randomly generated weights for each synapse. The corresponding inputs is summed to obtain first values of the hidden layer. These sums are smaller because they are not the final values for the hidden layer. To get the final value for the hidden layer, the activation feature must be applied. The role of an activation function is to introduce nonlinearity. An advantage of this is that the output is assigned from a range of 0 and 1, which makes it easier in the future to change the weights. The activation function of each hidden neuron is a so-called sigmoid function, a monotonically increasing function, that can be described with the following formulas:

$$
f: R \rightarrow[0,1] ; \lim x \rightarrow-\infty \text { with } f(x)=0 \wedge \lim x \rightarrow \infty \text { with } f(x)=1
$$

This feature has the advantage of being easy to calculate. A big disadvantage, however, is that it is not differentiable at the discontinuity and the derivative is everywhere 0 . This is a problem with the backpropagation algorithm because the derivative is included in the calculation of weight changes.

Therefore, a function is needed that approaches the binary threshold function as close as possible but is nevertheless differentiable and whose derivative is different from 0 . This requirement fulfills the so-called sigmoidal activation function, such as:

$$
f(x)=\frac{1}{1+e^{-x}}
$$

The logistics function has an easy-to-calculate derivation:

$$
\begin{aligned}
& f(x)=\frac{1}{1+e^{-x}}=\frac{e^{x}}{1+e^{x}} \\
& \frac{d}{d x} f(x)=\frac{e^{x} \cdot\left(1+e^{x}\right)-e^{x} \cdot e^{x}}{\left(1+e^{x}\right)}=\frac{e^{x}}{\left(1+e^{x}\right)}=f(x)(1-f(x))
\end{aligned}
$$

Thereafter, the matrix multiplication must be reused with another set of random weights to compute the value in the output layer. To normalize the output, the activation function is applied again last. The network also implies the algorithm for training the record "Backpropagation". At the beginning, all edge weights are randomly assigned. For all inputs in the training data set, the artificial network is activated, and its output is monitored. These outputs are compared to what we already know and expect, and the error "spreads" to the previous level. The error is marked, and the weight adjusted accordingly. This process is repeated until the output error is below the specified standard. After the above algorithm is completed, a learned artificial neural network is created. The network is considered as acceptable "new" input. It can be said that the artificial neural network learns from multiple samples (annotated data) and their errors (error propagation) [10]. After the first step forward transfer comes back propagation and weight updates, which explains the Backpropagation. All weights in the network are assigned randomly. Consider the node of the hidden layer. It is the weights associated with these nodes from the input layer. The neural network takes the first training sample as input.

\section{Experimental Results}

Fig. 3. shows the different results after training the data from the central router. The conclusions drawn from the graph serve as proof that a minimal statistical loss has been achieved with the used algorithms. Looking at the placement of the points, one can say that there is stable and steady growth. This reflects on the targeted result. Each next value is larger than the previous one. This is a result of the sigmoid function, which assumes only positive values. The Mark Denominator is an important number, and this is almost always different because of the weights used in the neural network. In addition, 
the sigmoid function also plays an important role here. Their advantage is that the negative inputs are strongly negative, and the zero inputs are close to zero in the tanh (Tanges Hyperbolicus) diagram. The function is differentiable and monotone while its derivative is not monotone. This feature makes it easier for the model to generalize or adapt to a variety of data and to distinguish between outputs. The main reason why the sigmoid function is used is that it exists between (0 to 1). Therefore, it is especially used for models where the probability of being output must be predicted. Since the probability of anything is only between 0 and 1 , Sigmoid is the right choice. In this work, the expected value is the mark denominator, which must necessarily be a positive number. As you can see from the graph, the values are positive and rising. The results are not far from each other. They are close. This observation speaks about a good implementation of the calculations in the neural network.

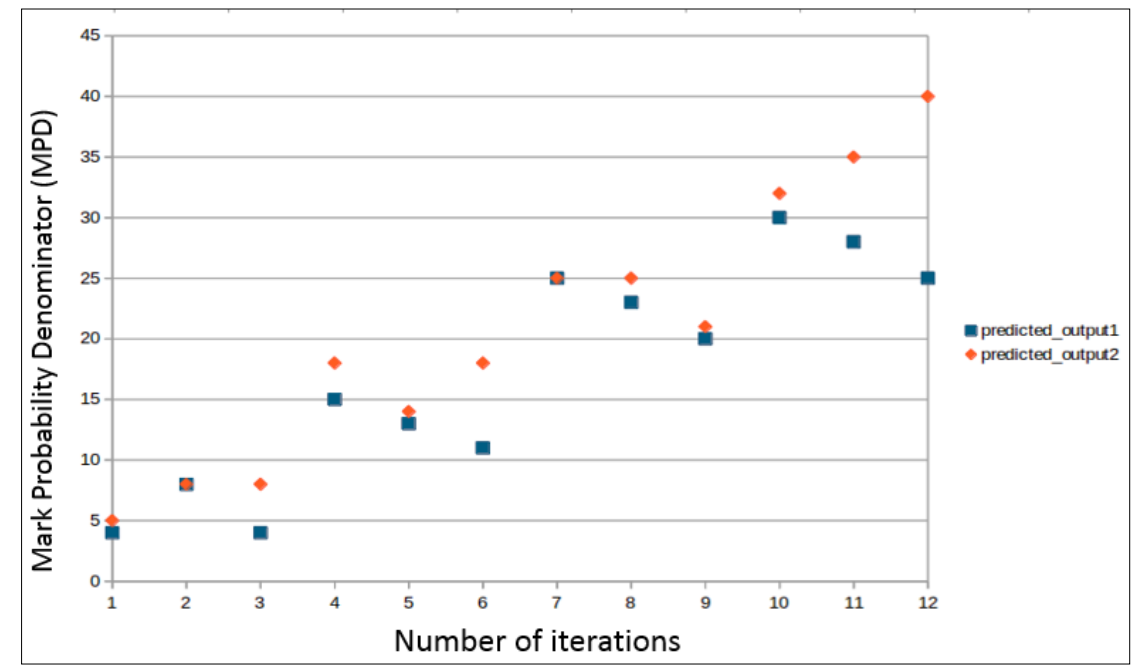

Fig. 3. Analysis of the trained data from MLP

The proof of this analysis of the reached data is explained by the many trainings of the neural network. The below Figures are showing the configuration of the New Router before and after implementing the new policy. In the New Router are configured only both classes Silver and Bronze.

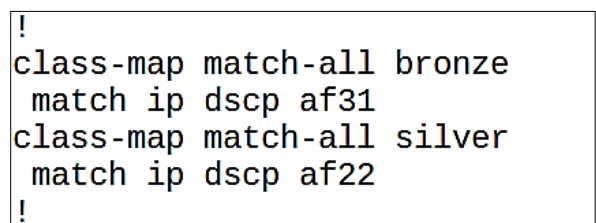

Fig. 4. Configuration in New Router before training the data set

As shown, there are two DSCP values implemented in the both classes. In class Bronze AF31 has a low drop probability and its equivalent precedence value is 011 - Flash. In class Silver AF22 has a medium drop probability and its precedence value is 010 - Intermediate.

In Central Router on Figure 5 is implemented the following configuration with WRED Algorithm.

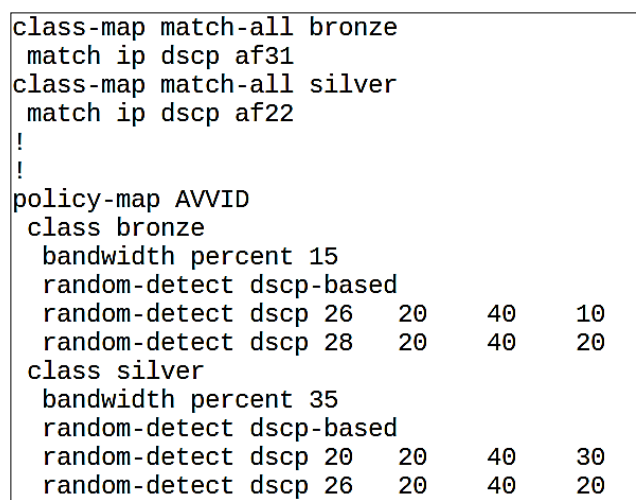

Fig. 5. The main configuration of Central Router 
One can see that, besides the implementation of both classes, there is also policy map with the name AVVID. For each class is applied the necessary DSCP value with its minimal and maximal threshold of the queue and the Mark Denominator. For example, the command random-detect dscp 20204030 describes as follows: dscp 20 is DSCP value 20, the second number 20 describes the minimal threshold, 40 is the maximal threshold and 30 is Mark Denominator. By 30 is meant that 1 of every 30 packets will be dropped if the average queue size is reached.

The API takes these values and passed them to the neural network in order to train them. An example how the API works is shown on Figure 6.

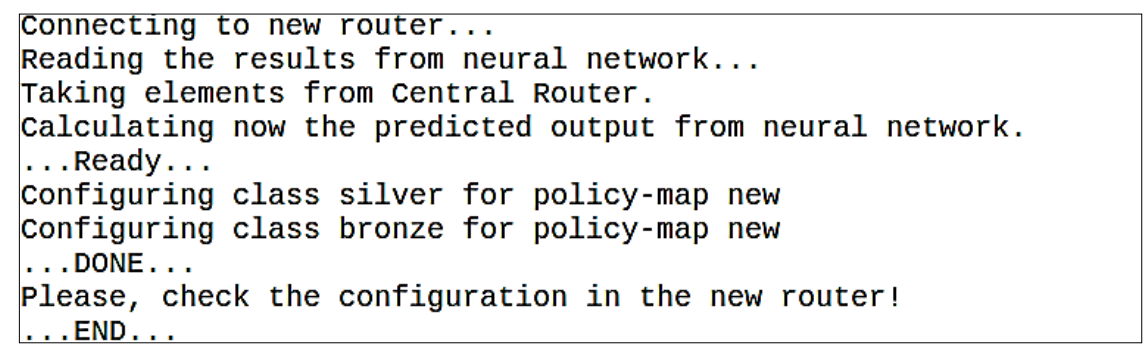

Fig. 6. Workflow of the API and implementing the results in the New Router

After finishing implementing one must check the results from the configuration in the New Router. On Figure 7 is shown what kind of policy must observe the New Router.

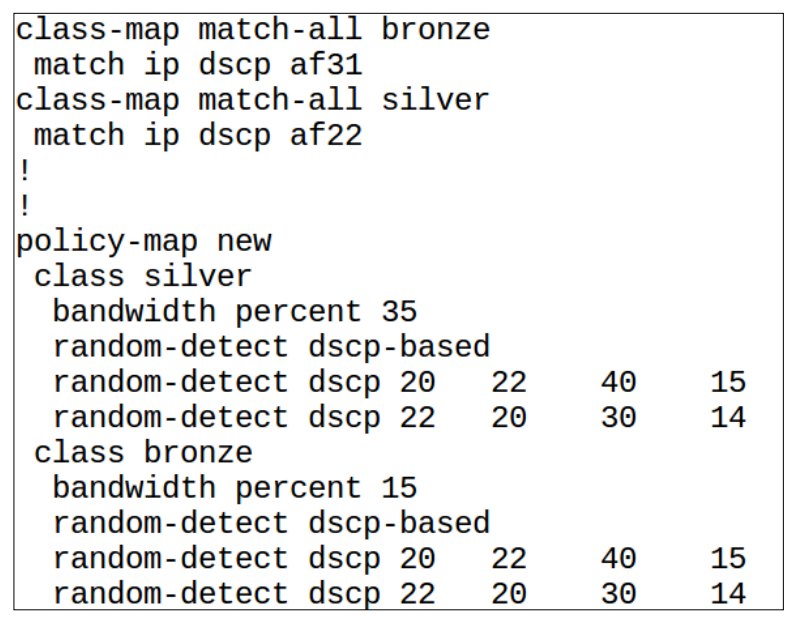

Fig. 7. After implementing the new policy in New Router

The difference in both routers is in the value of Mark Denominator. For example, in Central Router the value is 30 and in the New Router is 15. This depends on the minimal and maximal threshold of the queue.

\section{Conclusion}

Data exchange on the Internet happens through the constant transmission of packets between routers, between a router and a computer or between different smart devices. Therefore, there is the case that data packets can be lost. The problems can be for different reasons. Network overload is one of the most important basic terms when talking about data traffic. Typical effects include waiting times in the queue, packet loss, or blocking new connections. This must be somehow controlled. One of the most important point about it is the correct configuration of the network. With this work we solved this problem with creating an automated dynamic adaptation of QoS parameters in routers using specified QoS policies. The implemented API takes care of right transfer of the needed data between the router and the computer. The neural network used in this work showed a minimal statistical loss, achieved with the used algorithm. As shown in fig. 3 the graph speaks about a good implementation of the calculations in the neural network.

The above investigation showed an automated adaptation of additional network devices with specified QoS policies. This news will help a qualified person not constantly reviewing and changing the data. This means that the costs and the time are minimized. This automated customization will also affect the entire network connection and help with real-time systems by processing the data faster. This also reduces the latency and increases the data volume. The improved quality of data transmission is also an important point in this work and is also covered by the clarified experiment. 
This work also showed that WRED can avoid globalization issues that occur when tail-drop is used as a mechanism to prevent congestion on the router. For example, a global synchronization of TCP hosts may occur because packets are discarded at once. Global synchronization manifests itself when multiple TCP hosts reduce their transmission rates in response to dropping packets and then increase their transmission rates again when congestion is reduced.

WRED is composed of other techniques for avoiding congestion such as queue strategies. Attempts are made to expect and avoid overloading rather than controlling it as it occurs. One of the advantages of the WRED algorithm is the policy mappings that can be reused for multiple interfaces as needed. The thresholds for WRED and queue limits are specified and configured in policy maps. Once the thresholds are configured in a policy mapping, policy mapping can be applied to multiple interfaces, including interfaces with different bandwidths. This is especially useful when specifying the bandwidth for a class at an interface as a percentage of the total available bandwidth.

The purpose of this work for solving network congestion is to understand that the participation of neural networks is very important for proper configuration of the router. The presented variant for a data transfer plays a key role. Thus, the minimum threshold, the maximum threshold, and the mark denominator can be adjusted to determine the treatment packets receive by using the appropriate instruction. For example, the randomness ranking command can be used to determine the thresholds for an IP ranking.

\section{Future}

Having already understood why the implemented algorithm is a very good solution to the problem of network congestion, one can extend this architecture further. Future research could examine the use of automated software that takes care of the neural networks that are easily installed on different computers and could monitore the necessary configuration from the router. Beside that the implementation of a good database could lead to better graph results and investigation of new improvements. Minimizing the human work will help the easier implementation of the whole software.

The research on congestion avoidance will not end. There are several ways to adapt the data in the network to avoid such network overloads. More important here is the bottleneck management. Keeping traffic under constant control is a good example to minimize the abundance of data packets in the queue. Thus, the traffic can be facilitated and could increase the speed of data transfer.

\section{Acknowledgments}

I am especially indebted to Assoc. Prof. PhD. Ing. Irina Topalova and my Team Lead B. Eng. Dimitar Yanakiev, who have been supportive of my career goals and who worked actively to provide me with the protected academic time to pursue those goals. I am grateful to both with whom I have had the pleasure to work during this and other related projects.

\section{References}

[1] Samira Ch., Abdelouhab Z. and Jilali A. (25 July 2010). Identification and Prediction of Internet Traffic Using Artificial Neural Networks URL:

https://pdfs.semanticscholar.org/8e29/4e6c82a8df4d4e5a631bcc0f12a6af06a41c.pdf

[2] Macura, A., Missioni, E. and Marcovic, B. (2011). Quality of Service in Multimadia Computer Networks, Annals of DAAAM for 2011 \& Proceedings of the 22nd International DAAAM Symposium, Volume 22, No. 1, ISSN 1726- 9679 ISBN 978-3-901509-83-4.

[3] Vegesna, S. (2001). IP Quality of Service. Indianapolis, USA: Cisco Press

[4] Cisco Systems. (o.A.). Congestion Management Overview. Cisco IOS Quality of Service Solutions Configuration Guide: https://www.cisco.com/c/en/us/td/docs/ios/12_2/qos/configuration/guide/fqos_c/qcfconmg.pdf

[5] Sailu Reddy, A. A. (June 2009). TCP Performance Considerations. In HTTP: The Definitive Guide. O'Reilly Media.

[6] Ramakrishnan, K. (September 2001). The Addition of Explicit Congestion Notification (ECN) to IP. URL: https://tools.ietf.org/html/rfc3168

[7] Topalova, I. and Radoyska, P. (2018). Adjustment of the QoS Parameters on Routers with Neural Network Implementation, International Journal on Advances in Networks and Services, ISSN:1942-2644 vol. 11, no. 3 \& 4, pp. $143-151$.

[8] Gilbert, H. (2002). Quality of Service in a Cisco Networking Environment. ISBN-13: 978-0470844250. Wiley; 1 edition

[9] Cisco Systems. (o.A.). QoS Modular QoS Command-Line Interface Configuration Guide,Cisco IOS XE Fuji 16.7. URL: https://www.cisco.com/c/en/us/td/docs/ios-xml/ios/qos_mqc/configuration/xe-16-7/qos-mqc-xe-16-7book.pdf

[10] Scikit-learn developers. (2019). 1.17. Neural network models (supervised) / 1.17.1. Multi-layer Perceptron. URL scikit-learn.org: https://scikit-learn.org/stable/modules/neural_networks_supervised.html 